Article

\title{
Determination of the Electromagnetic Field on the Surface of the Beam Splitter of Laser Interferometer by Measuring the Displacements of Control Object Surfaces
}

\author{
Igor P. Miroshnichenko ${ }^{1, *}$, Ivan A. Parinov ${ }^{2}$, Shun-Hsyung Chang ${ }^{3}$ and Hung-Yu Wang ${ }^{3}$ \\ 1 Faculty of Technology Mashine, Don State Technical University, Rostov-on-Don 344000, Russia \\ 2 I.I. Vorovich Mathematics, Mechanics and Computer Sciences Institute, Southern Federal University, \\ Rostov-on-Don 344090, Russia; parinov_ia@mail.ru \\ 3 Nanzi Campus, National Kaohsiung University of Science and Technology, Kaohsiung 81143, Taiwan; \\ stephenshchang@me.com (S.-H.C.); hywang@kuas.edu.tw (H.-Y.W.) \\ * Correspondence: ipmir@rambler.ru; Tel.: +7-863-273-8543
}

Received: 24 September 2018; Accepted: 30 October 2018; Published: 2 November 2018

\begin{abstract}
The paper describes in detail the governing relationships for the electromagnetic field on the surface of the laser beam splitter of a laser interferometer. These expressions are intended for numerical simulation of measuring the displacements of control object surfaces by using a laser interferometer. We consider the cases of perpendicular and parallel polarizations of the radiation source. Moreover, the most relevant particular cases of constructing optical circuits of the interferometer in question are treated. The presented theoretical results have been used in numerical studies of the functional characteristics of promising contactless optical means of measuring displacements. The use of the described results has improved the quality and informativeness of the numerical simulation results of the measurement process of displacements by a laser interferometer.
\end{abstract}

Keywords: laser interferometer; displacement measurement; electromagnetic field; perpendicular polarization; parallel polarization

\section{Introduction, Purpose and Statement of the Problem}

At present, the development and use of contactless optical measuring instruments based on laser interferometers is one of the most relevant and promising scientific and technical areas. This is due to the need to improve the information content and accuracy of measurements of displacements of control surfaces. Such studies allow us to establish the features of the formation of defects in advanced materials and to perform diagnostics of the state of structural materials and goods. The measuring instruments [1-4], created on the base of a two-way laser interferometer with combined branches, are the most applicable and preferred for the solution of the pointed problems. The scheme of the laser interferometer is shown in Figure 1, where the beam splitter 1 with known amplitude transmission is located in a plane $S$, related to a associated Cartesian coordinate system $O x y z$. The reflector 2 is in a plane $S_{r}$ that is rotated relative to the plane $S$ by an angle $\alpha$. The reflector 2 is rigidly fixed on the surface of the control object and can perform linear $(\Delta h)$ and angular $(\Delta \alpha)$ displacements simultaneously with it. The beam splitter 1 receives coherent radiation from a point source of radiation in the form of an electric dipole located at the point $\mathrm{O}^{\prime}\left(x_{0}, y_{0}, z_{0}\right)$. The photodetectors for analyzing the interference field are located in the direction of reflection from the beam splitter of the source radiation, at a certain distance from the beam splitter corresponding to the Fresnel zone. This device allows us to determine 
the coordinates of the source of radiation from the interference pattern created, or if fixed, to find the movement of the interferometer reflector in space.

To determine the spatial displacement of the radiation source or reflector, it is necessary to define the change in the distribution of the electromagnetic field scattered by the device under consideration in the zone of observation of the interference pattern [5], i.e., in half-space $y>0$.

Using the tensor Green's function [6] with mixed Neumann-Dirichlet boundary conditions [7], and also taking into account the fact that the electromagnetic field has a transverse character and corresponds to the Maxwell equations, for its determination at the observation points it is sufficient to know only the tangential components of the electric field on the surface where the sources are located, i.e., on the beam splitter.

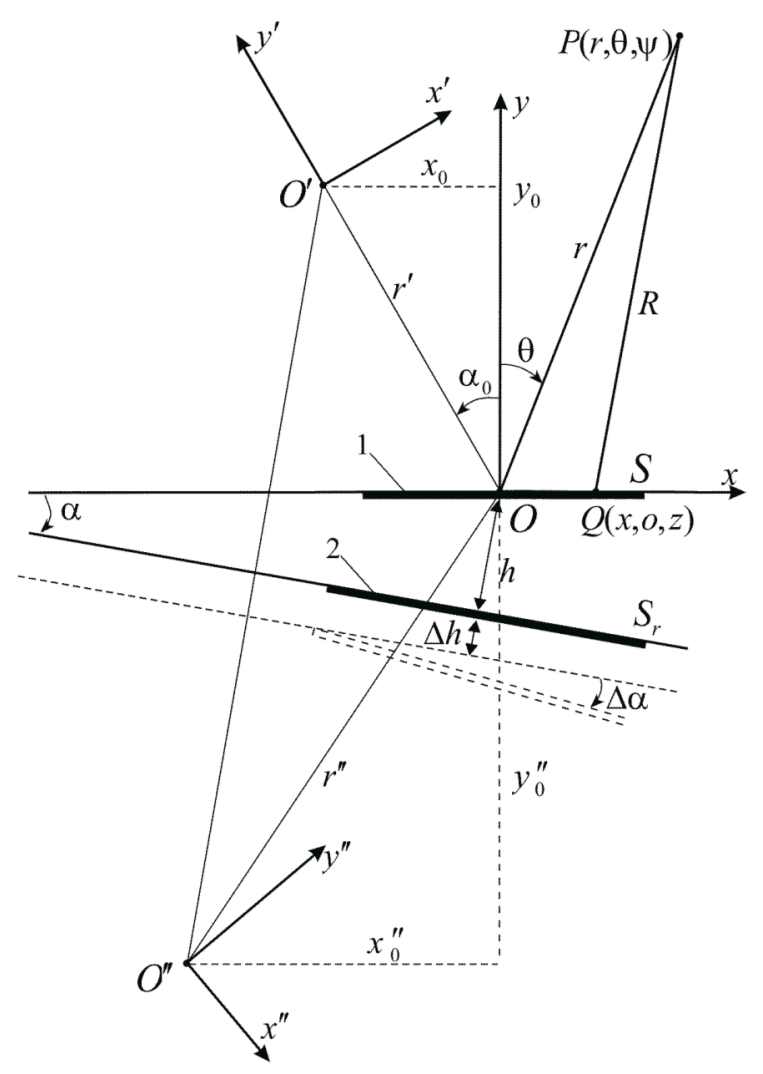

Figure 1. Circuits of interferometer under consideration.

To determine the tangential components of the electric field intensity vector $E$, the image method [8] is used, in accordance with which the total field is a superposition of the fields from the source at the point $O^{\prime}$ and the imaginary mirror source located at the point $O^{\prime \prime}$ taking into account the transmission of the beam splitter. Thus, the problem of determining the distribution of the intensity of the optical field in the area of the photodetector devices is solved successively in two stages, and initially, the field is determined on the beam splitter surface, and further, the field is determined in the area of the photodetector devices. At a given distribution of the intensity field in the area of photodetectors, it is possible to obtain displacements of the surface of the test object, uniquely associated with this area.

The aim of the work is to obtain the constitutive relations for determining the electromagnetic field on the surface of the beam splitter of the laser interferometer under consideration for the cases of perpendicular and parallel polarizations of the source radiation. These relations can be used for numerical simulation of the process of measuring the displacements of control object surfaces. 


\section{Determination of the Electromagnetic Field on the Beam Splitter Surface}

The relationships for the spherical components of the field of the source of electromagnetic radiation are sufficiently well-known [9-11]. If the beam splitter is flat, has a rectangular shape and is completely illuminated, then the field on its surface is convenient to express through the Cartesian components of the vector $E$, and in the case where the source field is a light spot on the beam splitter surface, it is more convenient to consider the cylindrical components of the vector $E$.

Let us consider the first case when the beam splitter is fully illuminated. For this, it is necessary, by transforming from spherical to Cartesian coordinates, to express the tangential components of this field on the beam splitter surface through the Cartesian components.

By solving the problem, it is convenient to additionally introduce auxiliary local Cartesian and associated spherical coordinate systems with origins at the points $O^{\prime}$ and $O^{\prime \prime}$. If the coordinates are written without strokes, then they correspond to the main system with the origin on the beam splitter surface at the point $O$, and the coordinates with one and two strokes refer, respectively, to the coordinate systems with the origin at the points $O^{\prime}$ and $O^{\prime \prime}$ (see Figure 1). Note that the coordinate system $O^{\prime} x^{\prime} y^{\prime} z^{\prime}$ is obtained from the system $O x y z$ by turning around the $z$-axis by an angle $\alpha_{0}$ in the positive direction and then parallel translation along the $x$-axis on the length $x_{0}$ and along the $y$-axis on the length $y_{0}$. The coordinate system $O^{\prime \prime} x^{\prime \prime} y^{\prime \prime} z^{\prime \prime}$ is obtained from the system $O^{\prime} x^{\prime} y^{\prime} z^{\prime}$ by mirroring it and then changing the direction of the $y$-axis to the opposite.

The Cartesian coordinate systems introduced are related to each other by the following equations:

$$
\begin{gathered}
x^{\prime}=\left(x+x_{0}\right) \cos \alpha_{0}+\left(y-y_{0}\right) \sin \alpha_{0} ; \\
y^{\prime}=-\left(x+x_{0}\right) \sin \alpha_{0}+\left(y-y_{0}\right) \cos \alpha_{0} ; \\
z^{\prime}=z ; \\
x^{\prime \prime}=\left(x+x^{\prime \prime}{ }_{0}\right) \cos \left(\alpha_{0}+2 \alpha\right)-\left(y+y^{\prime \prime}{ }_{0}\right) \sin \left(\alpha_{0}+2 \alpha\right) ; \\
y^{\prime \prime}=\left(x+x^{\prime \prime}{ }_{0}\right) \sin \left(\alpha_{0}+2 \alpha\right)+\left(y+y^{\prime \prime}{ }_{0}\right) \cos \left(\alpha_{0}+2 \alpha\right) ; \\
z^{\prime \prime}=z,
\end{gathered}
$$

where

$$
\begin{gathered}
x_{0}^{\prime \prime}=x_{0} \cos 2 \alpha+2 h \sin \alpha+y_{0} \sin 2 \alpha \\
y_{0}^{\prime \prime}=-x_{0} \sin 2 \alpha+2 h \cos \alpha+y_{0} \cos 2 \alpha .
\end{gathered}
$$

In the expressions (1)-(3), the values of the parameters $x_{0}, y_{0}, \alpha_{0}$ and $\alpha, h$ determine the position of the radiation source and the reflector in space (see Figure 1).

\section{Case of Perpendicular Polarization of Source Radiation}

If the dipole is parallel to the $z$-axis (see Figure 1), i.e., the electric field intensity vector lies in the plane perpendicular to the plane of incidence, then in the notations for the tangential components of the electric field intensity vector, we use the notation $\perp$.

Expressions that determine the relationships between Cartesian and spherical coordinates are known and have the following forms:

(i) for the basic coordinate system

$$
x=r \sin \theta \sin \psi ; \quad y=r \cos \theta ; \quad z=r \sin \theta \cos \psi ;
$$

Lamé coefficients are equal, respectively:

$$
h_{r}=1 ; \quad h_{\theta}=r ; \quad h_{\psi}=r \sin \theta ;
$$


(ii) for an auxiliary coordinate system with origin at the point $O^{\prime}$ :

$$
x^{\prime}=r^{\prime} \sin \theta^{\prime} \cos \psi^{\prime} ; \quad y^{\prime}=r^{\prime} \sin \theta^{\prime} \sin \psi^{\prime} ; \quad z^{\prime}=r^{\prime} \cos \theta^{\prime} ;
$$

(iii) for an auxiliary coordinate system with origin at the point $O^{\prime \prime}$ :

$$
x^{\prime \prime}=r^{\prime \prime} \sin \theta^{\prime \prime} \cos \psi^{\prime \prime} ; \quad y^{\prime \prime}=r^{\prime \prime} \sin \theta^{\prime \prime} \sin \psi^{\prime \prime} ; \quad z^{\prime \prime}=r^{\prime \prime} \cos \theta^{\prime \prime} .
$$

By substituting Expression (1) into Expression (6), and Expression (2) into Expression (7), we find the relationships of the basic Cartesian coordinate system $x, y, z$ and the auxiliary spherical ones:

(i) for the auxiliary coordinate system $O^{\prime} r^{\prime} \theta^{\prime} \psi^{\prime}$ :

$$
\begin{gathered}
r^{\prime} \sin \theta^{\prime} \cos \psi^{\prime}=\left(x+x_{0}\right) \cos \alpha_{0}+\left(y-y_{0}\right) \sin \alpha_{0} ; \\
r^{\prime} \sin \theta^{\prime} \sin \psi^{\prime}=-\left(x+x_{0}\right) \sin \alpha_{0}+\left(y-y_{0}\right) \cos \alpha_{0} ; \\
r^{\prime} \cos \theta^{\prime}=z
\end{gathered}
$$

(ii) for the auxiliary coordinate system $O^{\prime \prime} r^{\prime \prime} \theta^{\prime \prime} \psi^{\prime \prime}$ :

$$
\begin{aligned}
& r^{\prime \prime} \sin \theta^{\prime \prime} \cos \psi^{\prime \prime}=\left(x+x^{\prime \prime}{ }_{0}\right) \cos \left(\alpha_{0}+2 \alpha\right)-\left(y+y^{\prime \prime}{ }_{0}\right) \sin \left(\alpha_{0}+2 \alpha\right) ; \\
& r^{\prime \prime} \sin \theta^{\prime \prime} \sin \psi^{\prime \prime}=\left(x+x^{\prime \prime}{ }_{0}\right) \sin \left(\alpha_{0}+2 \alpha\right)+\left(y+y^{\prime \prime}{ }_{0}\right) \cos \left(\alpha_{0}+2 \alpha\right) ; \\
& r^{\prime \prime} \cos \theta^{\prime \prime}=z .
\end{aligned}
$$

From Equation (1), we obtain:

$$
y=y_{0}+x^{\prime} \sin \alpha_{0}+y^{\prime} \cos \alpha_{0} ; x=-x_{0}+x^{\prime} \cos \alpha_{0}-y^{\prime} \sin \alpha_{0} ; z=z^{\prime} .
$$

By substituting Expression (6) into Relationships (10), we obtain:

$$
\begin{gathered}
y=y_{0}+r^{\prime} \sin \theta^{\prime} \cos \psi^{\prime} \sin \alpha_{0}+r^{\prime} \sin \theta^{\prime} \sin \psi^{\prime} \cos \alpha_{0} ; \\
x=-x_{0}+r^{\prime} \sin \theta^{\prime} \cos \psi^{\prime} \cos \alpha_{0}-r^{\prime} \sin \theta^{\prime} \sin \psi^{\prime} \sin \alpha_{0} ; \\
z=r^{\prime} \cos \theta^{\prime} .
\end{gathered}
$$

Similarly to Expression (11), we have

$$
\begin{gathered}
y=y_{0}^{\prime \prime}-r^{\prime \prime} \sin \theta^{\prime \prime} \cos \psi^{\prime \prime} \sin \left(\alpha_{0}+2 \alpha\right)+r^{\prime \prime} \sin \theta^{\prime \prime} \sin \psi^{\prime \prime} \cos \left(\alpha_{0}+2 \alpha\right) ; \\
x=-x_{0}^{\prime \prime}+r^{\prime \prime} \sin \theta^{\prime \prime} \cos \psi^{\prime \prime} \cos \left(\alpha_{0}+2 \alpha\right)+r^{\prime \prime} \sin \theta^{\prime \prime} \sin \psi^{\prime \prime} \sin \left(\alpha_{0}+2 \alpha\right) ; \\
z=r^{\prime \prime} \cos \theta^{\prime \prime} .
\end{gathered}
$$

To obtain the relationships connecting the components of the vector $\boldsymbol{E}$ in the selected coordinate systems, we apply the well-known formula relating the components of vectors in different orthogonal coordinate systems [12]:

$$
\widetilde{E}_{k} \widetilde{h}_{k}=\frac{\partial x^{m}}{\partial \widetilde{x}^{k}} E_{m} h_{m}
$$

where $\widetilde{E}_{k}$ and $E_{m}$ are the physical components of vector $\boldsymbol{E}$ in the first and second coordinate systems, respectively, $\widetilde{h}_{k}$ and $h_{m}$ are the corresponding metric Lamé coefficients.

By assuming the "first" coordinate system is Cartesian, the "second" coordinate system is spherical $O^{\prime} r^{\prime} \theta^{\prime} \psi^{\prime}$ or $O^{\prime \prime} r^{\prime \prime} \theta^{\prime \prime} \psi^{\prime \prime}$ and using Expression (13), we can obtain relationships for the components of the vector $E^{\prime}$ of source field $O^{\prime}$ or $O^{\prime \prime}$ in the Cartesian coordinate system through the spherical components of this vector. 
For the incident field, we obtain

$$
\begin{aligned}
E_{x \perp}^{\prime} h_{x} & =\frac{\partial r^{\prime}}{\partial x} E_{r^{\prime}}^{\prime} h_{r^{\prime}}+\frac{\partial \theta^{\prime}}{\partial x} E_{\theta^{\prime}}^{\prime} h_{\theta^{\prime}}+\frac{\partial \psi^{\prime}}{\partial x} E_{\psi^{\prime}}^{\prime} h_{\psi^{\prime}} \\
E_{z \perp}^{\prime} h_{z} & =\frac{\partial r^{\prime}}{\partial z} E_{r^{\prime}}^{\prime} h_{r^{\prime}}+\frac{\partial \theta^{\prime}}{\partial z} E_{\theta^{\prime}}^{\prime} h_{\theta^{\prime}}+\frac{\partial \psi^{\prime}}{\partial z} E_{\psi^{\prime}}^{\prime} h_{\psi^{\prime}} \\
E_{y \perp}^{\prime} h_{y} & =\frac{\partial r^{\prime}}{\partial y} E_{r^{\prime}}^{\prime} h_{r^{\prime}}+\frac{\partial \theta^{\prime}}{\partial y} E_{\theta^{\prime}}^{\prime} h_{\theta^{\prime}}+\frac{\partial \psi^{\prime}}{\partial y} E_{\psi^{\prime}}^{\prime} h_{\psi^{\prime}}
\end{aligned}
$$

In accordance with Expression (13), the tangential components of the electric field intensity vector on the surface $y=0$ can be written in the form:

$$
E_{x \perp}^{\prime}=\frac{\partial x}{\partial \theta^{\prime}} \cdot \frac{1}{h_{\theta^{\prime}}} E_{\theta^{\prime}} ; E_{z \perp}^{\prime}=\frac{\partial z}{\partial \theta^{\prime}} \cdot \frac{1}{h_{\theta^{\prime}}} E_{\theta^{\prime}} .
$$

Here $E_{x \perp}^{\prime}$ and $E_{z \perp}^{\prime}$ are present via $E_{\theta^{\prime}}^{\prime}$, since the other components of the dipole radiation in the Fresnel zone $E_{\psi^{\prime}}^{\prime}=E_{r^{\prime}}^{\prime}=0$.

From Equations (11), we obtain

$$
\begin{gathered}
\frac{\partial x}{\partial \theta^{\prime}}=\left(r^{\prime} \cos \psi^{\prime} \cos \alpha_{0}-r^{\prime} \sin \psi^{\prime} \sin \alpha_{0}\right) \cos \theta^{\prime}=r^{\prime} \cos \left(\psi^{\prime}+\alpha_{0}\right) \cos \theta^{\prime} \\
\frac{\partial z}{\partial \theta^{\prime}}=-r^{\prime} \sin \theta^{\prime} .
\end{gathered}
$$

By substituting Expression (16) and corresponding Lamé coefficients into Expression (15), we obtain

$$
E_{x \perp}^{\prime}=\cos \left(\psi^{\prime}+\alpha_{0}\right) \cos \theta^{\prime} \cdot E_{\theta^{\prime}}^{\prime} ; E_{z \perp}^{\prime}=-\sin \theta^{\prime} \cdot E_{\theta^{\prime}}^{\prime} .
$$

If the radiation source is removed from the beam splitter by a distance corresponding to the Fresnel zone and further, then in the spherical coordinate system $O^{\prime} r^{\prime} \theta^{\prime} \psi^{\prime}$, the field on the surface $y=0$ can be represented only by the transverse components of the vectors $E^{\prime}$ and $H^{\prime}$ [9-11] as

$$
H_{\psi^{\prime}}^{\prime}=-\frac{i k I^{\prime} l}{4 \pi r^{\prime}} \sin \theta^{\prime} \cdot e^{i k r^{\prime}}=-A_{0} \frac{i k}{r^{\prime}} \sin \theta^{\prime} \cdot e^{i k r^{\prime}} ; E_{\theta^{\prime}}^{\prime}=A_{0} \frac{i k^{2}}{\omega \varepsilon r^{\prime}} \sin \theta^{\prime} \cdot e^{i k r^{\prime}} .
$$

Other components of the vectors $E^{\prime}$ and $H^{\prime}$ can be neglected, then, assuming $A_{0}=1$, we have:

$$
H_{\psi^{\prime}}^{\prime}=-\frac{i k}{r^{\prime}} \sin \theta \cdot e^{i k r^{\prime}} ; E_{\theta^{\prime}}^{\prime}=-\frac{i k^{2}}{\omega \varepsilon r^{\prime}} \sin \theta \cdot e^{i k r^{\prime}},
$$

where $r^{\prime}=\sqrt{\left(x+x_{0}\right)^{2}+y_{0}^{2}+z^{2}}$, because $y=0$.

Then Expression (17) transform to

$$
E_{x \perp}^{\prime}=\frac{i k^{2}}{\omega \varepsilon} \cdot \frac{\cos \theta^{\prime} \sin \theta^{\prime} \cos \left(\psi^{\prime}+\alpha_{0}\right)}{r^{\prime}} e^{i k r^{\prime}} ; E_{z \perp}^{\prime}=-\sin \theta^{\prime} \cdot \frac{i k^{2}}{\omega \varepsilon} \cdot \frac{\sin \theta^{\prime}}{r^{\prime}} e^{i k r^{\prime}} .
$$

From Relationship (8), we obtain:

$$
\begin{aligned}
& \cos \theta^{\prime}=\frac{z}{r^{\prime}}=\frac{z}{\sqrt{\left(x+x_{0}\right)^{2}+\left(y-y_{0}\right)^{2}+z^{2}}} \\
& \tan \psi^{\prime}=\frac{-\left(x+x_{0}\right) \sin \alpha_{0}+\left(y-y_{0}\right) \cos \alpha_{0}}{\left(x+x_{0}\right) \cos \alpha_{0}+\left(y-y_{0}\right) \sin \alpha_{0}} .
\end{aligned}
$$


From Expression (21), we obtain:

$$
\sin \theta^{\prime}=\sqrt{1-\frac{z^{2}}{\left(r^{\prime}\right)^{2}}}=\frac{\sqrt{\left(x+x_{0}\right)^{2}+\left(y-y_{0}\right)^{2}}}{\sqrt{\left(x+x_{0}\right)^{2}+\left(y-y_{0}\right)^{2}+z^{2}}} .
$$

From Expression (8), we obtain:

$$
\cos \psi^{\prime}=\frac{\left(x+x_{0}\right) \cos \alpha_{0}+\left(y-y_{0}\right) \sin \alpha_{0}}{r^{\prime} \sin \theta^{\prime}} .
$$

From Expression (23), we obtain:

$$
\left(r^{\prime}\right)^{2} \sin ^{2} \theta^{\prime}=\left(x+x_{0}\right)^{2}+\left(y-y_{0}\right)^{2} .
$$

Then, instead of Relationship (24), we obtain:

$$
\cos \psi^{\prime}=\frac{\left(x+x_{0}\right) \cos \alpha_{0}+\left(y-y_{0}\right) \sin \alpha_{0}}{\sqrt{\left(x+x_{0}\right)^{2}+\left(y-y_{0}\right)^{2}}} .
$$

From Expression (8), we obtain:

$$
\sin \psi^{\prime}=\frac{-\left(x+x_{0}\right) \sin \alpha_{0}+\left(y-y_{0}\right) \cos \alpha_{0}}{r^{\prime} \sin \theta^{\prime}} .
$$

By substituting Expression (25) into Relationship (27), we obtain:

$$
\sin \psi^{\prime}=\frac{-\left(x+x_{0}\right) \sin \alpha_{0}+\left(y-y_{0}\right) \cos \alpha_{0}}{\sqrt{\left(x+x_{0}\right)^{2}+\left(y-y_{0}\right)^{2}}} .
$$

Let us write the Cartesian components of the vectors $E^{\prime}$ and $H^{\prime}$ in the basic coordinate system Oxyz; with this aim, we substitute Expressions (21), (23), (26), (28), into (20) and taking into account that $y=0$, we obtain:

$$
E_{x \perp}^{\prime}=\frac{i k^{2}}{\omega \varepsilon} \cdot \frac{z\left(x+x_{0}\right)}{\left(r^{\prime}\right)^{3}} e^{i k r^{\prime}} ; E_{z \perp}^{\prime}=-\frac{i k^{2}}{\omega \varepsilon} \cdot \frac{\left(x+x_{0}\right)^{2}+y_{0}^{2}}{\left(r^{\prime}\right)^{3}} e^{i k r^{\prime}},
$$

where $r^{\prime}=\sqrt{\left(x+x_{0}\right)^{2}+y_{0}^{2}+z^{2}}$.

Similarly, one can obtain expressions for the tangent field components from the imaginary source:

$$
E_{x \perp}^{\prime \prime}=\frac{i k^{2}}{\omega \varepsilon} \cdot \frac{z\left(x+x_{0}^{\prime \prime}\right)}{\left(r^{\prime \prime}\right)^{3}} e^{i k r^{\prime \prime}} ; E_{z \perp}^{\prime \prime}=-\frac{i k^{2}}{\omega \varepsilon} \cdot \frac{\left(x+x_{0}^{\prime \prime}\right)^{2}+y_{0}^{\prime \prime 2}}{\left(r^{\prime \prime}\right)^{3}} e^{i k r^{\prime \prime}},
$$

where $r^{\prime \prime}=\sqrt{\left(x+x^{\prime \prime} 0\right)^{2}+y^{\prime \prime} 0^{2}+z^{2}}$.

\section{Case of Parallel Polarization of Source Radiation}

If the dipole is parallel to the $x^{\prime}$-axis, i.e., the vector of the electric field intensity is parallel to the plane of incidence, then in the notations for the tangential components of the electric field intensity vector we use the notation $\|$.

Expressions that determine the relationships between Cartesian and spherical coordinates are known and have the following forms:

(i) for the basic coordinate system, they are found by Formulae (4); 
(ii) for an auxiliary coordinate system with origin at the point $O^{\prime}$ :

$$
x^{\prime}=r^{\prime} \cos \theta^{\prime} ; y^{\prime}=r^{\prime} \sin \theta^{\prime} \cos \psi^{\prime} ; z^{\prime}=r^{\prime} \sin \theta^{\prime} \sin \psi^{\prime} ;
$$

(iii) for an auxiliary coordinate system with origin at the point $O^{\prime \prime}$ :

$$
x^{\prime \prime}=r^{\prime \prime} \cos \theta^{\prime \prime} ; y^{\prime \prime}=r^{\prime \prime} \sin \theta^{\prime \prime} \cos \psi^{\prime \prime} ; z^{\prime \prime}=r^{\prime \prime} \sin \theta^{\prime \prime} \sin \psi^{\prime \prime} .
$$

By substituting Relationship (31) into Expression (10), we obtain:

$$
\begin{gathered}
y=y_{0}+r^{\prime} \cos \theta^{\prime} \sin \alpha_{0}+r^{\prime} \sin \theta^{\prime} \cos \psi^{\prime} \cos \alpha_{0} ; \\
x=-x_{0}+r^{\prime} \cos \theta^{\prime} \cos \alpha_{0}-r^{\prime} \sin \theta^{\prime} \cos \psi^{\prime} \sin \alpha_{0} ; \\
z=r^{\prime} \sin \theta^{\prime} \sin \psi^{\prime} .
\end{gathered}
$$

Similarly to Expression (33), we obtain:

$$
\begin{gathered}
y=y^{\prime \prime}{ }_{0}-r^{\prime \prime} \cos \theta^{\prime \prime} \sin \left(\alpha_{0}+2 \alpha\right)+r^{\prime \prime} \sin \theta^{\prime \prime} \cos \psi^{\prime \prime} \cos \left(\alpha_{0}+2 \alpha\right) ; \\
x=-x^{\prime \prime}{ }_{0}+r^{\prime \prime} \cos \theta^{\prime \prime} \cos \left(\alpha_{0}+2 \alpha\right)+r^{\prime \prime} \sin \theta^{\prime \prime} \cos \psi^{\prime \prime} \sin \left(\alpha_{0}+2 \alpha\right) ; \\
z=r^{\prime \prime} \sin \theta^{\prime \prime} \sin \psi^{\prime \prime} .
\end{gathered}
$$

From Relationship (33), we obtain:

$$
\frac{\partial x}{\partial \theta^{\prime}}=-r^{\prime} \sin \theta^{\prime} \cos \alpha_{0}-r^{\prime} \cos \theta^{\prime} \cos \psi^{\prime} \sin \alpha_{0} ; \frac{\partial z}{\partial \theta^{\prime}}=r^{\prime} \cos \theta^{\prime} \sin \psi^{\prime} .
$$

By substituting Expression (35) and the corresponding Lamé coefficients into relationships (15), we obtain:

$$
E_{x}=-\left(\sin \theta^{\prime} \cos \alpha_{0}+\cos \theta^{\prime} \cos \psi^{\prime} \sin \alpha_{0}\right) \cdot E_{\theta^{\prime}} ; E_{z}=\cos \theta^{\prime} \sin \psi^{\prime} \cdot E_{\theta^{\prime}} .
$$

By substituting Expression (18) into Relationships (36), we have

$$
E_{x}=-\left(\sin \theta^{\prime} \cos \alpha_{0}+\cos \theta^{\prime} \cos \psi^{\prime} \sin \alpha_{0}\right) \cdot \frac{i k^{2}}{\omega \varepsilon} \cdot \frac{\sin \theta^{\prime}}{r^{\prime}} e^{i k r^{\prime}} ; E_{z}=\cos \theta^{\prime} \sin \psi^{\prime} \cdot \frac{i k^{2}}{\omega \varepsilon} \cdot \frac{\sin \theta^{\prime}}{r^{\prime}} e^{i k r^{\prime}}
$$

From Relationships (31) we find:

$$
\cos \psi^{\prime}=\frac{y^{\prime}}{r^{\prime} \sin \theta^{\prime}} ; \sin \psi^{\prime}=\frac{z^{\prime}}{r^{\prime} \sin \theta^{\prime}} ; \cos ^{2} \psi^{\prime}+\sin ^{2} \psi^{\prime}=1=\frac{\left(y^{\prime}\right)^{2}+\left(z^{\prime}\right)^{2}}{\left(r^{\prime} \sin \theta^{\prime}\right)^{2}} .
$$

Then

$$
\sin \theta^{\prime}=\frac{\sqrt{\left(y^{\prime}\right)^{2}+\left(z^{\prime}\right)^{2}}}{r^{\prime}} .
$$

By substituting expressions for $y^{\prime}$ and $z^{\prime}$ from Expression (1) into Relationship (39), we obtain:

$$
\sin \theta^{\prime}=\frac{\sqrt{\left[-\left(x+x_{0}\right) \sin \alpha_{0}+\left(y-y_{0}\right) \cos \alpha_{0}\right]^{2}+z^{2}}}{\sqrt{\left(x+x_{0}\right)^{2}+\left(y-y_{0}\right)^{2}+z^{2}}} .
$$

Then we substitute Expressions (1) and (40) into Relationship (38):

$$
\cos \psi^{\prime}=\frac{-\left(x+x_{0}\right) \sin \alpha_{0}+\left(y-y_{0}\right) \cos \alpha_{0}}{\sqrt{\left[-\left(x+x_{0}\right) \sin \alpha_{0}+\left(y-y_{0}\right) \cos \alpha_{0}\right]^{2}+z^{2}}} ;
$$




$$
\sin \psi^{\prime}=\frac{z^{\prime}}{\sqrt{\left[-\left(x+x_{0}\right) \sin \alpha_{0}+\left(y-y_{0}\right) \cos \alpha_{0}\right]^{2}+z^{2}}} .
$$

Now, by using Expression (31), we obtain:

$$
\cos \theta^{\prime}=\frac{x^{\prime}}{r^{\prime}}=\frac{\left(x+x_{0}\right) \cos \alpha_{0}+\left(y-y_{0}\right) \sin \alpha_{0}}{\sqrt{\left(x+x_{0}\right)^{2}+\left(y-y_{0}\right)^{2}+z^{2}}} .
$$

Let us write the Cartesian components of the vectors $E^{\prime}$ and $H^{\prime}$ in the basic coordinate system Oxyz; with this aim, we substitute Expressions (40), (41), (42), (43), into (37) and taking into account that $y=0$, we obtain:

$$
\begin{gathered}
E_{x \|}^{\prime}=-i \frac{k^{2}}{\omega \varepsilon} \cdot \frac{\left[\left(x+x_{0}\right) \sin \alpha_{0}+y_{0} \cos \alpha_{0}\right] y_{0}+z^{2} \cos \alpha_{0}}{\left(r^{\prime}\right)^{3}} e^{i k r^{\prime}} \\
E_{z \|}^{\prime}=i \frac{k^{2}}{\omega \varepsilon} \cdot \frac{\left[\left(x+x_{0}\right) \cos \alpha_{0}-y_{0} \sin \alpha_{0}\right] z}{(r \prime)^{3}} e^{i k r \prime},
\end{gathered}
$$

where $r^{\prime}=\sqrt{\left(x+x_{0}\right)^{2}+y_{0}^{2}+z^{2}}$.

Similarly, one can obtain expressions for the tangent field components from the imaginary source:

$$
\begin{gathered}
E_{x / /}^{\prime \prime}=-i \frac{k^{2} e^{i k r^{\prime \prime}}}{\omega \varepsilon}\left[\frac{-\left(x+x_{0}^{\prime \prime}\right) \sin \left(\alpha_{0}+2 \alpha\right) y_{0}^{\prime \prime}}{\left(r^{\prime \prime}\right)^{3}}+\frac{y_{0}^{\prime \prime 2} \cos \left(\alpha_{0}+2 \alpha\right)+z^{2} \cos \left(\alpha_{0}+2 \alpha\right)}{\left(r^{\prime \prime}\right)^{3}}\right] ; \\
E_{z / /}^{\prime \prime}=i \frac{k^{2} e^{i k r^{\prime \prime}}}{\omega \varepsilon}\left[\frac{\left(x+x_{0}^{\prime \prime}\right) \cos \left(\alpha_{0}+2 \alpha\right) z}{\left(r^{\prime \prime}\right)^{3}}+\frac{y_{0}^{\prime \prime} \sin \left(\alpha_{0}+2 \alpha\right) z}{\left(r^{\prime \prime}\right)^{3}}\right],
\end{gathered}
$$

where $r^{\prime \prime}=\sqrt{\left.\left(x+x^{\prime \prime} 0\right)^{2}+\left(y^{\prime \prime}\right)_{0}\right)^{2}+z^{2}}$.

By using the Expressions (29), (30), (44), and (45), it is possible to determine the components of the electric field intensity vector of the incident or reflected wave tangential to the beam splitter surface for a given polarization of the source radiation.

The total field on the beam splitter surface for each polarization can be written, using the expressions:

$$
E_{x}=E_{x}^{\prime}+T_{x}(x, z) E_{x}^{\prime \prime} ; E_{z}=E_{z}^{\prime}+T_{z}(x, z) E_{z}^{\prime \prime} .
$$

Here $T(x, z)$ is the amplitude transmission of the beam splitter, it can be different for different polarizations of the irradiating light [13].

Thus, it can be noted that the relationships are obtained for determining the field distribution on the beam splitter surface of the laser interferometer under consideration, which is represented by the tangential components of the electric field intensity vector $E_{x}$ and $E_{y}$ expressed in the Cartesian coordinates of the basic system Oxyz, taking into account the polarization of the radiation source and the transmittance of the beam splitter.

\section{Calculated Relationships for Particular Cases of Optical Circuits}

If a circular diaphragm with diameter $2 a$ is the source of radiation, whose center is located at the point $\mathrm{O}^{\prime}\left(x_{0}, y_{0}, 0\right)$ (see Figure 2), and the normal to the diaphragm aperture makes an angle $\alpha_{0}$ with the $y$-axis, then in Relationships (17) and (36), the quantities $E_{\theta^{\prime}}^{\prime}$ and $E_{\psi^{\prime}}^{\prime}$ for the case of polarization in question will be determined by the expressions:

$$
\begin{aligned}
E_{\theta^{\prime} \|}^{\prime} & =-i \frac{\exp \left(i k r^{\prime}\right)}{r^{\prime}} \cdot \frac{a \sin \psi^{\prime}}{\sin \theta^{\prime}} J_{1}\left(k a \sin \theta^{\prime}\right) ; E_{\psi^{\prime} \|}^{\prime}=-i \frac{\exp \left(i k r^{\prime}\right)}{r^{\prime}} \cdot \frac{a \cos \theta^{\prime} \cos \psi^{\prime}}{\sin \theta^{\prime}} J_{1}\left(k a \sin \theta^{\prime}\right) ; \\
E_{\theta^{\prime} \perp}^{\prime} & =-i \frac{\exp \left(i k r^{\prime}\right)}{r^{\prime}} \cdot \frac{a \cos \psi^{\prime}}{\sin \theta^{\prime}} J_{1}\left(k a \sin \theta^{\prime}\right) ; E_{\psi^{\prime} \perp}^{\prime}=-i \frac{\exp \left(i k r^{\prime}\right)}{r^{\prime}} \cdot \frac{a \cos \theta^{\prime} \sin \psi^{\prime}}{\sin \theta^{\prime}} J_{1}\left(k a \sin \theta^{\prime}\right),
\end{aligned}
$$


where $a$ is the diaphragm radius, $J_{1}$ is the Bessel function of the first order.

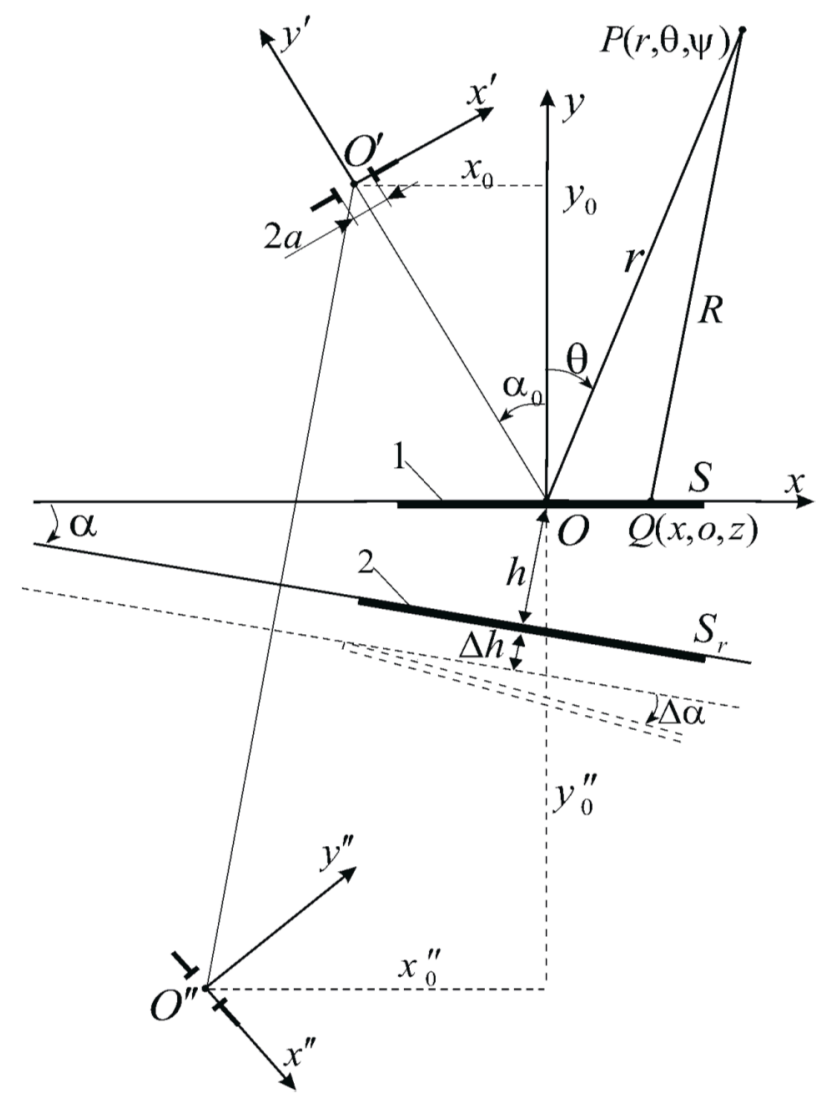

Figure 2. Special case of optical circuits (the source of radiation is the diaphragm).

Similar relationships can be obtained for a mirror diaphragm, where instead of the coordinates of the system denoted by a single stroke, it is necessary to write coordinates with two strokes. By substituting Relationship (47) in Expressions (17) and (36), and carrying out transformations similar to those given above, one can obtain expressions for calculating the field on the beam splitter surface.

In the case when the beam splitter is not completely illuminated (represents itself a round "spot", described on the surface of the beam splitter by cylindrical coordinates $\rho$ and $\varphi$ ), it is convenient to represent the source field by the cylindrical components of the vector $E_{\rho}$ and $E_{\varphi}$. On the surface $y=0$, the expressions for these components have the form:

$$
\begin{gathered}
E_{\rho}=\frac{\bar{r}^{\prime}}{\bar{r}^{\prime \prime}}\left[\sin \varphi \sin \alpha_{0}+\frac{\bar{y}^{\prime}\left(\rho+x_{0} \sin \varphi\right)}{\left(\bar{r}^{\prime}\right)^{2}}\right] E_{\theta^{\prime}}^{\prime}+\frac{\bar{r}^{\prime \prime}\left(-x_{0} \cos \alpha_{0}+y_{0} \sin \alpha_{0}\right) \cos \varphi}{\rho^{2} \cos ^{2} \varphi+(\bar{x})^{2}} E_{\psi^{\prime}}^{\prime} ; \\
E_{\varphi}=\frac{\bar{r}^{\prime} \cos \varphi}{\bar{r}^{\prime \prime}}\left[\sin \alpha_{0}+\frac{\bar{y}^{\prime} x_{0}}{\left(\bar{r}^{\prime}\right)^{2}}\right] E_{\theta^{\prime}}^{\prime}+\frac{\bar{r}^{\prime \prime}\left(\rho \cos \alpha_{0}+x_{0} \sin \varphi \cos \alpha_{0}-y_{0} \sin \varphi \sin \alpha_{0}\right)}{\rho^{2} \cos ^{2} \varphi+(\bar{x})^{2}} E_{\psi^{\prime}}^{\prime},
\end{gathered}
$$

where $\bar{r}^{\prime}=\sqrt{\rho^{2}+2 \rho x_{0} \sin \varphi+x_{0}^{2}+y_{0}^{2}} ; \bar{x}^{\prime}=\left(\rho \sin \varphi+x_{0}\right) \cos \alpha_{0}-y_{0} \sin \alpha_{0}$;

$$
\bar{y}^{\prime}=-\left(\rho \sin \varphi+x_{0}\right) \sin \alpha_{0}-y_{0} \cos \alpha_{0} ; \bar{r}^{\prime}=\sqrt{\left(\bar{r}^{\prime}\right)^{2}-(\bar{y} \prime)^{2}} .
$$

The same relationships can be obtained by expanding the vector $E$ written in a spherical coordinate system associated with a mirror diaphragm. To do this, we need to replace the notations with one stroke by two strokes in Expressions (48) and (49), and replace $x_{0}, y_{0}, \alpha_{0}$ by values of $x_{0}^{\prime \prime}, y_{0}^{\prime \prime},-\left(\alpha_{0}+2 \alpha\right)$ from Expression (3), respectively. 
The presented relationships have been used in the original software [14-18] for numerical studies of the functional characteristics of promising contactless means designed to measurements of optical displacements $[19,20]$. The use of the relations described in the paper has made it possible to improve the quality and informativeness of the numerical simulation of the process of measuring displacements by a laser interferometer [19].

\section{Conclusions}

In the paper, the constitutive relations are obtained for the determination of the electromagnetic field on the surface of the beam splitter of the laser interferometer for the cases of perpendicular and parallel polarization of the source radiation. The relationships are also obtained for particular cases of optical schemes for the interferometer under consideration. These relationships allow us to perform numerical simulation of the process of measuring the displacements of control object surfaces using a laser interferometer.

The described results have been used in numerical studies of the functional characteristics of promising contactless optical means for measuring displacements. Their use has improved the quality and informativeness of the numerical simulation of the measurement process of displacements by a laser interferometer.

The obtained relationships can also be used in theoretical and numerical modeling and for justification of new contactless optical interference measurement methods and advanced optical means for measuring displacements of control object surfaces.

Author Contributions: M.I.P.-original draft preparation and determination of electromagnetic field, M.I.P., P.I.A.—validation of research methods, S.-H.C.—conceptualization, H.-Y.W.- review and editing.

Funding: The work was supported by the grant of Russian Foundation for Basic Research, No. 16-08-00740, Ministry of Education and Science of the Russian Federation: projects Nos. 3.5378.2017/VU (organization of the implementation of researches), 9.9770.2017/BCh (the basic part of the state task), Ministry of Science and Technology of the Republic of China (Grant No. MOST 105-2923-E-992-302-MY3).

Acknowledgments: The equipment of SFedU and DSTU was used.

Conflicts of Interest: The authors declare no conflict of interest.

\section{References}

1. Miroshnichenko, I.P.; Parinov, I.A.; Rozhkov, E.V.; Serkin, A.G.; Sizov, V.P. Novel Test Means and Techniques Based on Optical Interferometry and Acoustic Emission to Study Displacements of Object Surfaces and Damage of High-Temperature Superconductive Tapes and Related Composites. In Piezoelectrics and Related Materials: Investigations and Applications; Nova Science Publishers: Hauppauge, NY, USA, 2012; pp. 239-282.

2. Miroshnichenko, I.P.; Parinov, I.A.; Rozhkov, E.V.; Sizov, V.P.; Shevtsov, V.A. Novel Optical Means for Measurement of Displacements of Surfaces of the Control Objects at Diagnostics of Materials and Goods. In Physics and Mechanics of New Materials and Their Applications; Nova Science Publishers: Hauppauge, NY, USA, 2013; pp. 145-154.

3. Miroshnichenko, I.P.; Parinov, I.A.; Rozhkov, E.V.; Chang, S.-H. Mathematical Models, Program Software, Technical and Technological Solutions for Measurement of Displacements of the Control Object Surfaces by Laser Interferometer. In Advanced Materials—Manufacturing, Physics, Mechanics and Applications, Springer Proceedings in Physics; Springer International Publishing: Cham, Switzerland, 2016; Volume 175, pp. 341-356.

4. Miroshnichenko, I.P.; Parinov, I.A.; Wu, J.-K.; Hong, W.-L.; Yeh, M.-Y. Novel optical interference means to measure small linear and angular displacements of control object surfaces. In Advanced Materials-Techniques, Physics, Mechanics and Applications, Springer Proceedings in Physics; Springer International Publishing: Cham, Switzerland, 2017; Volume 193, pp. 591-605.

5. Born, M.; Volf, E. Osnovy Optiki; Nauka: Moscow, Russia, 1979; p. 719. (In Russian)

6. Morse, P.M.; Feshbach, H. Methods of Theoretical Physics; Inostrannaya Literatura: Moscow, Russia, 1960; Volume 2, p. 896. (In Russian) 
7. Kovalenko, N.V. On tensor Green function in boundary problems of electrodynamics. In Antennye Reshetki; Rostov State University Press: Rostov-on-Don, Russia, 1971; pp. 17-33. (In Russian)

8. Morse, P.M.; Feshbach, H. Methods of Theoretical Physics; Inostrannaya Literatura: Moscow, Russia, 1960; Volume 1, p. 930. (In Russian)

9. Felsen, L.B.; Marcuvitz, N. Radiation and Scattering of Waves; Mir: Moscow, Russia, 1978; Volume 1, p. 552. (In Russian)

10. Markov, G.T.; Petrov, B.M.; Grudinskaya, G.P. Electrodinamika i Rasprostranenie Radiovoln; Sovetskoe Radio: Moscow, Russia, 1979; p. 373. (In Russian)

11. Landau, L.F.; Lifshitz, E.M. The Classical Theory of Fields; Nauka: Moscow, Russia, 1967; p. 460. (In Russian)

12. Singe, J.L. Relativity - The General Theory; North-Holland Publishing Company: Amsterdam, The Netherlands, 1960; p. 505.

13. Stroke, G.J. An Introduction to Coherent Optics and Holography; Academic Press: Cambridge, MA, USA, 1966; p. 348.

14. Miroshnichenko, I.P. Program for Determining the Intensity of the Optical Field of epy Interference Pattern (Case of Perpendicular Polarization). Certificate of State Registration of the Computer Program No. 2014614501 RF, 2014. Available online: http:/ /www1.fips.ru/Archive/EVM/2014/2014.05.20/DOC/ RUNW/000/002/014/614/501/document.pdf (accessed on 31 October 2018). (In Russian)

15. Miroshnichenko, I.P. The Program for Determining the Intensity of the Optical Field of the Interference Pattern (the Case of Parallel Polarization). Certificate of State Registration of the Computer Program No. 2014614502 RF, 2014. Available online: http:/ / www1.fips.ru/Archive/EVM/2014/2014.05.20/DOC/ RUNW/000/002/014/614/502/document.pdf (accessed on 31 October 2018). (In Russian)

16. Miroshnichenko, I.P.; Shevtsov, V.A. Determination of the Intensity of the Optical Field of the Interference Pattern Produced by a Laser Two-Way Interferometer with a Combined Branches. Certificate of State Registration of the Computer Program No. 2014662261 RF, 2014. Available online: http://www1.fips. ru/Archive/EVM/2014/2014.12.20/DOC/RUNW/000/002/014/662/261/document.pdf (accessed on 31 October 2018). (In Russian)

17. Miroshnichenko, I.P.; Parinov, I.A. Determination of the Intensity of the Optical Field of the Interference Pattern Produced by a Laser Interferometer for a Beam Splitter in the Form of a Phase Sinusoidal Grating with a Uniform Period. Certificate of State Registration of the Computer Program No. 2015610921 RF, 2015. Available online: http:/ / www1.fips.ru/Archive/EVM/2015/2015.02.20/DOC/RUNW/000/002/015/610/ 921/ document.pdf (accessed on 31 October 2018). (In Russian)

18. Miroshnichenko, I.P.; Parinov, I.A. Determination of the Intensity of the Optical Field of the Interference Pattern Produced by a Laser Interferometer for a Beam Splitter in the Form of a Phase Zone Plate. Certificate of State Registration of the Computer Program No. 2015611078 RF, 2015. Available online: http:/ /www1. fips.ru/Archive/EVM/2015/2015.02.20/DOC/RUNW /000/002/015/611/078/document.pdf (accessed on 31 October 2018). (In Russian)

19. Miroshnichenko, I.P.; Parinov, I.A. Scientific Ground of a New Optical Device for Contactless Measurement of the Small Spatial Displacements of Control Object Surfaces. IOP Conf. Ser. Mater. Sci. Eng. 2017, 209, 012054. [CrossRef]

20. Miroshnichenko, I.P.; Parinov, I.A. Development of a New Optical Measuring Set-Up. IOP Conf. Ser. Mater. Sci. Eng. 2018, 374, 012052. [CrossRef]

(C) 2018 by the authors. Licensee MDPI, Basel, Switzerland. This article is an open access article distributed under the terms and conditions of the Creative Commons Attribution (CC BY) license (http://creativecommons.org/licenses/by/4.0/). 